\title{
Urgences
}

\section{Les manœuvres attentives}

\section{Jean-Marc Bélanger}

Numéro 29, octobre 1990

\section{Éclats d'œuvre}

URI : https://id.erudit.org/iderudit/025600ar

DOI : https://doi.org/10.7202/025600ar

Aller au sommaire du numéro

Éditeur(s)

Urgences

\section{ISSN}

0226-9554 (imprimé)

1927-3924 (numérique)

Découvrir la revue

Citer ce document

Bélanger, J.-M. (1990). Les manœuvres attentives. Urgences, (29), 38-44.

https://doi.org/10.7202/025600ar d'utilisation que vous pouvez consulter en ligne.

https://apropos.erudit.org/fr/usagers/politique-dutilisation/ 


\section{MANCEUVRES ET PIĖGES}




\section{Les manœuvres attentives Jean-Marc Bélanger}

La question de l'œuvre... Pour l'aborder, j'ai voulu éviter les voies le plus souvent empruntées, ignorer Mallarmé, Blanchot. Autant que possible, j'ai cherché à reprendre l'œuvre à zéro, à réinventer un parcours. Suivant mes humeurs, suivant les rumeurs de l'œuvre, je me suis lancé sur des pistes moins familières, convaincu de pouvoir, au besoin, tracer mes propres sentes; déjà préjugeant de l'œuvre. Bientôt, entre un dictionnaire et un roman de Jacques Poulin, un essai philosophique de Michel Guérin et certaines considérations esthétiques d'Umberto Eco, toutes mes certitudes se sont défilées. Ce que j'appelais à moi dans l'espoir de constituer un énoncé théorique cohérent ne faisait que s'entasser en vrac sur le papier. Au bord du désœuvrement, j'escomptais encore la jouissance prochaine de quelque privilège attaché à mon extra-territorialité théorique et, d'illusion en illusion, l'alignement préalablement imaginé restait hors d'atteinte.

Il a fallu que dans l'intimité de ma conscience l'écriture reprenne son fief pour qu'enfin je me retrouve à pied d'œuvre avec, tout autour de moi, des matériaux épars, disponibles. 
Maintenant, nous le savons: l'ouverture est la condition même de la jouissance esthétique, et toute forme dont on peut jouir pour ce qu'elle est dotée d'une valeur esthétique est * ouverte". Elle l'est, nous l'avons vu, alors même que l'artiste tend à une communication univoque et non ambiguë.

Reste que l'étude des cuvres ouvertes contemporaines met en lumiére autre chose, une intention d'ouverture explicite et portée à son extrême limite; cette ouverture ne se base plus seulement sur les caractères propres de l'objet sur sa composition, mais sur les éléments me̊me qui y entrent. ${ }^{1}$

Parmi les concepts en usage dans les différents domaines de l'art, certains semblent échapper à toute précise définition malgré tous les efforts déployés pour atteindre à une quelconque scientificité. Bien que nombre de notions courantes aient été précisées, bien qu'une multitude de nouveaux concepts aient été définis au fil des dernières décennies, on n'a pu encore décrire, entre autres, la spécificité du littéraire ni l'universalité de l'art. C'est très souvent en vain qu'on a aussi tenté de repousser les limites de notre ignorance en créant des néologismes ou en accolant des étiquettes rutilantes à de vieilles idées (toutefois, on a pu ainsi parler avec aise et d'abondance de sujets telle la littérarité du nouveau roman). Tels efforts de particularisation, sans même pallier à l'inaccessibilité d'une certaine essence, mènent invariablement à l'abîme car, de la différence envisagée ainsi, extérieurement, ne peut résulter que, fatals, le manque ou l'excès. Parmi cette mouvance où, fluide, le sens se transforme, se transvase, certains concepts sont encore repoussés aux confins de plusieurs champs notionnels. Ainsi en est-il de l'œuvre qu'on envisage tant à partir de l'art qu'à partir de la morale. L'œuvre qui file en tous genres. L'œuvre, singulière et plurielle; d'usages dérivés en locutions figées elle - et, 
40

même, il - divague en tous sens, jusqu'à se perdre au large, jusqu'à sombrer littéralement dans le tourbillon métonymique: «ignorer Mallarmé, Blanchot». Dans l'œuvre, même envisagée d'un strict point de vue littéraire, se répercutent des échos maints. Telle chronique imprécision provoque l'instabilité du littéraire et assigne l'écrivain à une éternelle reprise en sous-œuvre.

Considérant les propos d'Umberto Eco mis en exergue à cette section des Manœuvres et décrivant l'œuvre comme ouverte, on observera qu'à l'autre bout du langage perdure le silence et qu'en admettant l'ouverture de l'œuvre on n'atteint pas encore à son essence. À la rigueur, on accepte de s'ouvrir soi-même davantage à ce qui dans l'œuvre nous sollicite ou, mieux, on interroge une pratique sous le nouvel angle suggéré. Au pire cependant, on fait de l'idée d'ouverture le critère d'un jugement esthétique.

Mais tout cela n'est que fiction de l'œuvre car un peu moins de trente années se sont écoulées depuis la parution de L'ceuvre ouverte et on peut déjà se demander ce qu'il advient de l'édifice prédicatif d'Eco. Peut-être cette construction, comme tant d'autres, n'a-t-elle pu qu'éprouver les fondements toujours invisibles de l'œuvre. En littérature, on a depuis jugé qu'une tendance trop appuyée à l'ouverture de l'œuvre vers d'infinies possibilités d'interprétation risquait de jeter celle-ci dans l'abîme d'une irréductible ambiguitté. Ce faisant, l'on n'a fait que prendre pour réalité l'expression diverse d'une idée-limite. Depuis, on assiste à un important déploiement d'efforts pour imposer une nouvelle tendance qui se donne comme un idéal reconquis sous le fard de son vocable: la nouvelle lisibilité. Plus que jamais peut-être, dans la seule désignation d'un courant littéraire, on s'acoquine ici au langage. Parlant d'une * nouvelle* lisibilité, on en affirme une ancienne. On reprend ici la formule du "même amélioré" pourtant déjà éculée par la publicité. On se réclame d'une continuité par l'idée de retour incluse dans l'épithète "nouvelle en même temps qu'on marque son opposition à tout ce qui ne serait pas lisible en jouant de l'opposition irréductible entre lisibilité et illisibilité. Le principe global est simple: entre le nouvel et l'ancien, tout est évacué. Dans le vide ainsi obtenu, il y a l'espace suffisant à l'érection d'une critique qui peut prendre toutes les allures d'un rappel à l'ordre. 
On comprend bien, au bout du compte, qu'il n'y eut jamais d'ancienne, voire de nouvelle lisibilité. Le seul rappel à l'ordre possible est rappel à l'œuvre qui, de tous temps, ne sait se soumettre à d'autres injonctions que les siennes propres, aussi diverses que le travail maint qui s'y investit et s'y récupère. 
Toute cuvre est une pensée, non pas exprimée, mais imprimée. ${ }^{2}$

Créer, c'est savoir qu'on crée; ce qui ne veut pas dire qu'on soit en mesure d'expliquer pourquoi ni comment. ${ }^{3}$

Il n'y a plus désormais, parmi les Mancuvres, ni sarcasme ni ironie. Le temps n'est plus à la cabriole sémantique par laquelle les mots des autres se retournent contre eux. La perspective est ici toute différente. La piste qui se dessine maintenant part de la page avec ses blancs vertiges et traverse l'œuvre entière dans son accomplissement. Cependant, il ne faut pas voir, dans la description de ce parcours, la promesse de quelque ultime révélation. Pas plus ici qu'ailleurs ne saurait être évitée l'angoisse du non-sens. La voix de l'écrivain en est pleine; si limpide qu'elle semble, elle vacille encore entre le cri de la bête et le silence du néant.

L'écrivain s'inscrit dans la distance, pour oublier son humaine condition, pour déjouer l'issue fatale. Il élabore, à partir du langage, des constructions analogiques pour saisir le sens et le soumettre à l'œuvre. Partant, celle-ci prendra mainte forme: celle d'un court poème qui, laissant sur la table de l'écrivain cent pages de notes inutilisées, s'achèvera en fines ciselures, celle encore d'un roman historique étoffé des résultats d'innombrables recherches.

Michel Guérin affirme qu'en raison du travail qu'elle implique, l'œuvre, comme toute tâche humaine, * tombe sous le coup de la nécessité * ${ }^{4}$. Toutefois, le travail de l'œuvre échappe seul à l'écueil de la répétition et l'énergie qu'il exige est seule à n'être pas dépensée en pure perte. Ce qui dans l'œuvre vient contrer la nécessité, c'est le fait que celle-ci soit voulue, jusqu'à l'acharnement, jusque dans l'indifférence en face de la vie, de la mort. En cela, l'œuvre trouve son essence.

Ainsi, l'écrivain considère l'accomplissement de l'œuvre comme nécessaire et poursuit telle fin. En même temps,

2 Michel Guérin, Qu'est-ce qu'une ceuvre, coll. * Le Génie du philosophe ", Arles, Actes Sud, 1986, p. 71.

3 lbid., p. 61.

4 Ibid., p. 29. 
échappant à la nécessité, le travail investi dans l'œuvre participe désormais à sa croissance. Le cercle de l'œuvre est établi. Celle-ci se révèle à la fois produit et production, créée et création. En somme, l'écrivain pourrait être vu comme un joueur d'échecs solitaire, acharné à la formation d'un adversaire capable de le battre. Suivant la logique de cette comparaison, le maître-d'œuvre apparaît comme celui qui, humble et volontaire, cherche la leçon d'œuvre.

Au fil de sa réflexion, Michel Guérin introduit l'idée de la contemplation en distinguant le jugement contemplatif (de celui qui reçoit l'œuvre), de la volonté contemplative (de celui qui crée). Celui qui reçoit vient participer à l'œuvre en reconnaissant et confirmant son autorité. Le créateur, pour sa part, s'absorbe dans la contemplation de la nécessité même. L'un et l'autre sont essentiels à l'œuvre qui, pour atteindre à l'existence, doit être deux fois traduite.

Ainsi parfaitement indifférente au temps et entièrement réductible au temps, l'œuvre, maintenue dans sa forme, solide dans son intégrité, recueillie en soi, inscrit également à son actif les interprétations successives qui paraissant s'éloigner d'elle, voire la trahir, portent témoignage de sa consistance et alors qu'on les supposerait attentatoires à sa pureté, se soumettent, en réalité, au commandement qui émane d'elle; il se ramasse pour qui en saisit toute la force, dans l'aphorisme: créer la création. 
Je ne sais pas pourquoi mais, tout au long, la musique a porté cette réflexion. Peut-être même l'a-t-elle portée jusqu'au vertige.

Je songe par exemple à Jessy Norman interprétant Les quatre derniers chants de Richard Strauss, écrits sur des textes de Herman Hesse et Joseph von Eichendorf. L'œuvre réalisée en 1948, un an avant le décès du compositeur, poursuit une réflexion grave en même temps que sereine sur la mort.

Je songe aussi au Carmina burana de Carl Orff. À partir d'une suite de poèmes lyriques latins des douzième et treizième siècles, le musicien crée une atmosphère sonore unique, à la fois grandiose et profondément troublante.

Non, je ne crois pas qu'il soit possible à l'esprit de saisir l'œuvre. Du moins l'entreprise me semble-t-elle paradoxale car, dans l'œuvre, il m'apparaît y avoir déjà de l'esprit, jadis enlevé à la matière, ou évadé, pour tout le temps.

- As-tu trouvé ce que tu cherchais ? demanda-t-elle.

- Non, dis-je, mais ça ne fait rien. Parfois les réponses ne veulent pas venir. Parfois on ne trouve que des questions.

- As-tu encore de la peine?

- Un peu.

- Qu'est-ce que tu vas faire?

- Je vais faire comme j'avais dit: je vais essayer d'écrire la plus belle histoire d'amour qui ait jamais été écrite.

La petite se mit à sourire.

- Et les questions dont tu viens de parler, tu vas les mettre dans ton histoire? demanda-t-elle.

- Bien sûr, dis-je. 5

5 Jacques Poulin, Le vieux chagrin, Arles/Montreal, Actes Sud/Leméac, 1989, p. 154. 